\title{
Genesis
}

Manuscrits - Recherche - Invention

\section{Des éditions génétiques haïtiennes : pourquoi ? pour qui ? comment?}

Le cas de Dézafi/Les Affres d'un défi de Frankétienne

\section{Jean Jonassaint}

\section{OpenEdition}

\section{Journals}

Édition électronique

URL : http://journals.openedition.org/genesis/607

DOI : 10.4000/genesis.607

ISSN : 2268-1590

Éditeur :

Presses universitaires de Paris Sorbonne (PUPS), Société internationale de génétique artistique littéraire et scientifique (SIGALES)

\section{Édition imprimée}

Date de publication : 30 octobre 2011

Pagination : 79-92

ISBN : 978-2-84050-804-5

ISSN : $1167-5101$

Référence électronique

Jean Jonassaint, «Des éditions génétiques haïtiennes : pourquoi ? pour qui ? comment? 》, Genesis [En ligne], 33 | 2011, mis en ligne le 23 octobre 2013, consulté le 19 avril 2019. URL : http:// journals.openedition.org/genesis/607 ; DOI : 10.4000/genesis.607 


\title{
Des éditions génétiques haïtiennes : pourquoi ? pour qui ? comment ? Le cas de Dézafi/Les Affres d'un défi de Frankétienne
}

\author{
Jean Jonassaint
}

$\mathrm{R}$ eprenant les questions de Sartre dans Qu'est-ce que la littérature? (1948), avant toute entreprise d'édition critique ou génétique d'œuvres haïtiennes, nous devons nous demander : Qu'est-ce qu'une édition génétique? Pourquoi des éditions génétiques d'œuvres haïtiennes? Pour qui ? Mais aussi ce qui semble être moins manifeste dans l'essai de Sartre : Comment? Un comment qui, dans un premier temps, renvoie aux fondements mêmes de la critique génétique : les divers procès de production d'une œuvre, du désir vague ou intense d'écrire ou de faire un livre jusqu'à l'ouvrage imprimé, commenté. Dans un deuxième temps non moins important, mais que nous écartons ici, ce "comment» renvoie également aux moyens matériels (sources de financement) et intellectuels (équipes de chercheurs compétents et motivés) à mobiliser pour reconstituer la genèse d'un texte. Par ailleurs, la tâche première de définir les démarches génétiques sera également écartée. Nous nous concentrerons plutôt sur les tenants et aboutissants d'éventuelles éditions génétiques de Dézafi/Les Affres d'un défi de Frankétienne1, qui sont les textes choisis pour cette exploration du comment écrivent les Haïtiens.

\section{Pourquoi ces textes ?}

Sans conteste, ces œuvres sont des plus représentatives des littératures haïtiennes en français et en haïtien, comme le prouve entre autres leur exceptionnelle fortune critique universitaire et populaire, tant nationale qu'internationale. À titre d'exemple, rappelons le commentaire de Chamoiseau et Confiant dans leurs Lettres créoles : « [...] c'est en 1975 avec la publication du premier roman en créole haïtien, Dézafi, que la littérature créole $[\ldots]$ va trouver ses lettres de noblesse. [...] C'est la révolution. L'écrit en créole accède d'un coup à ce que la littérature mondiale possédait à l'époque de plus moderne, de plus audacieux et de plus talentueux, à savoir le Nouveau Roman ${ }^{2} »$. Par ailleurs, avec Dézafi et sa version française Les Affres d'un défi, Frankétienne donne l'ensemble contemporain le plus emblématique de la déchirure langagière qui travaille les productions haïtiennes. D'abord publié à Port-au-Prince, Dézafi (1975) est un récit haïtien, en haïtien, pour les Haïtiens, de la résurrection des zombis par le sel, mais le texte français publié à Port-au-Prince en 1979 , puis à Paris en $2000^{3}$, vise un double lectorat national et transnational, haïtien et francophone. Ce diptyque est aussi symptomatique de l'incessante quête d'un double lectorat dont Cric ? Crac ! de Georges Sylvain $(1901)^{4}$ offre le modèle le plus complexe (voir fig. 1). Partant d'un hypotexte archiconnu, les fables de

1. Ce travail s'inscrit dans le cadre d'un projet plus vaste d'éditions génétiques d'œuvres haïtiennes au sein de l'équipe «Manuscrit francophone » de 1'ITEM. Dans un premier temps, il devait inclure les récits de René Depestre, écrivain du dehors dont les principaux titres - Alléluia pour une femme-jardin : récits (1981); Hadriana dans tous mes rêves (1988) ; Éros dans un train chinois : nouvelles (1990) - ont été publiés dans la «collection blanche » de Gallimard. Des contraintes diverses n'ont malheureusement pas permis que ces textes soient vraiment abordés ici.

2. Patrick Chamoiseau et Raphaël Confiant, Lettres créoles. Tracées antillaises et continentales de la littérature, 1655-1975, Paris, Hatier, 1991, p. 173.

3. Voir Frankétienne, Les Affres d'un défi, Port-au-Prince, Imprimerie Henri Deschamps, 1979 (Paris, Jean-Michel Place, 2000 ; La Roque d'Anthéron, Vents d'ailleurs, 2010) ; Dézafi, Port-au-Prince, Éditions Fardin, 1975 (Châteauneuf-le-Rouge, Vents d'ailleurs, 2002). Pour une bio-bibliographie détaillée, voir : <www.lehman.cuny.edu/ile.en.ile/ paroles/franketienne.html>.

4. Georges Sylvain, Cric ? Crac! Les fables de La Fontaine racontées par un montagnard haïtien et transcrites en vers créoles, Paris, Ateliers haïtiens, 1901. 
La Fontaine qui sont aussi hypertextes, Sylvain donne sur la même page divisée en deux parties égales une double traduction, en haut celle en haïtien d'une fable (canonique française), en bas une traduction auctoriale, en français, de l'adaptation haïtienne du texte de La Fontaine ; en fin de volume des notes sur ses stratégies de traduction en fonction des poétiques différentes des deux langues. Il s'agit, avant la lettre, d'un exemple ancien d'autoédition critique dans une perspective génétique. Ce qui est systématique et manifeste dans Cric ? Crac! n'est pas moins à l'œuvre dans tout un corpus narratif haïtien. En effet, le métadiscours des procès de traduction ${ }^{5}$, donc des stratégies d'écriture, traverse les textes haïtiens de l'ouverture de La Famille des Pitite-Caille de Justin Lhérisson $(1905)^{6}$, exposant la pertinence d'une narratique proprement nationale, à la clôture de Éros dans un train chinois : nouvelles, de René Depestre (1990) sur un « glossaire érotique » auquel renvoient des astérisques fourmillant dans les textes. Ces récits, comme d'autres de Depestre, forment un corpus fort révélateur de la tension géopolitique qui traverse les productions haïtiennes, notamment dans leur quête de consécration nationale et transnationale.

Le lecteur attentif aura compris le pourquoi de ces textes de Frankétienne et de leurs analyses génétiques. Elles actualisent de manière exemplaire deux des traits les plus pertinents des récits haïtiens : l'autotraduction manifeste ou latente imposée par la coprésence de deux langues apparentées sous-tendant des répertoires divers, et les exigences d'un lectorat double, sinon multiple qui détermine, sinon surdétermine, les stratégies d'écriture, notamment l'omniprésence ou la prolifération dans les textes de procès de traduction qui sont d'abord des explications pour l'Autre. Mais une question demeure : qu'est-ce qu'une approche génétique d'œuvres haïtiennes peut apporter à l'analyse de la genèse des textes?

\section{Pourquoi une génétique des œuvres haïtiennes ?}

Une première réponse se dégage des esquisses d'analyse du Cric ? Crac ! de Sylvain ou du diptyque Dézafi/Les Affres d'un défi de Frankétienne : l'approche génétique de tels textes éclairerait de manière inédite la traduction auctoriale. La démarche de ces auteurs, contrairement à celles des Beckett, Green, Nabokov généralement étudiées ${ }^{7}$, n'est pas la résultante de choix individuels, elle est surdéterminée sur le plan d'une littérature nationale, conçue à partir de deux langues distinctes mais proches, pour un double lectorat. Ici s'entrevoit un vaste champ comparatif de choix traductologiques, donc de réécritures de mêmes fragments discursifs, d'un écrivain à un autre, ou même d'un texte à un autre qui est loin d'être soupçonné par une certaine critique européenne, comme le rappellent ces propos d'Antoine Berman dans L'Épreuve de l'étranger (1984) cités par Oustinoff : "Pour nous, les autotraductions sont des exceptions, tout comme le fait qu'un écrivain - pensons à Conrad et à Beckett choisisse une autre langue que la sienne propre ${ }^{8}$. » Pourtant, c'est bien là une commune condition des écrivains francophones du Sud, et une non moins commune mesure des œuvres haïtiennes particulièrement : quel que soit le texte, il en existe, du moins en fragment, un premier état (auctorial) transcrit ou non, autrement dit travaillé mentalement ou scripturalement dans une autre langue. Si les ouvrages ci-dessus mentionnés sont exemplaires, offrant deux états imprimés dans deux langues, il reste que l'ensemble du corpus narratif haïtien, en français notamment, peut jusqu'à un certain point être lu comme hypertexte des textes des auteurs ou d'un répertoire oral indigène ou indigénisé, comme le récit du pacte fatal avec le diable pour s'enrichir sur lequel se construit Le Crayon de Dieu de Philippe Thoby-Marcelin et Pierre Marcelin (1952).

5. Sur ce concept emprunté à Abdelkebir Khatibi, voir Jean Jonassaint, Des romans de tradition haïtienne: sur un récit tragique, Paris et Montréal, L'Harmattan et Cidihca, 2002, p. 114-130.

6. Justin Lhérisson, La Famille des Pitite-Caille, Paris, Typographie Firmin-Didot, 1905. Sur ce romancier, voir J. Jonassaint, Des romans de tradition haïtienne, op. cit.

7. Voir entre autres Michaël Oustinoff, Bilinguisme d'écriture et autotraduction : Julien Green, Samuel Beckett, Vladimir Nabokov (Paris, L'Harmattan, 2001), ouvrage qui ouvre des voies fort pertinentes pour la génétique des traductions auctoriales.

8. M. Oustinoff, op. cit., p. 35. 

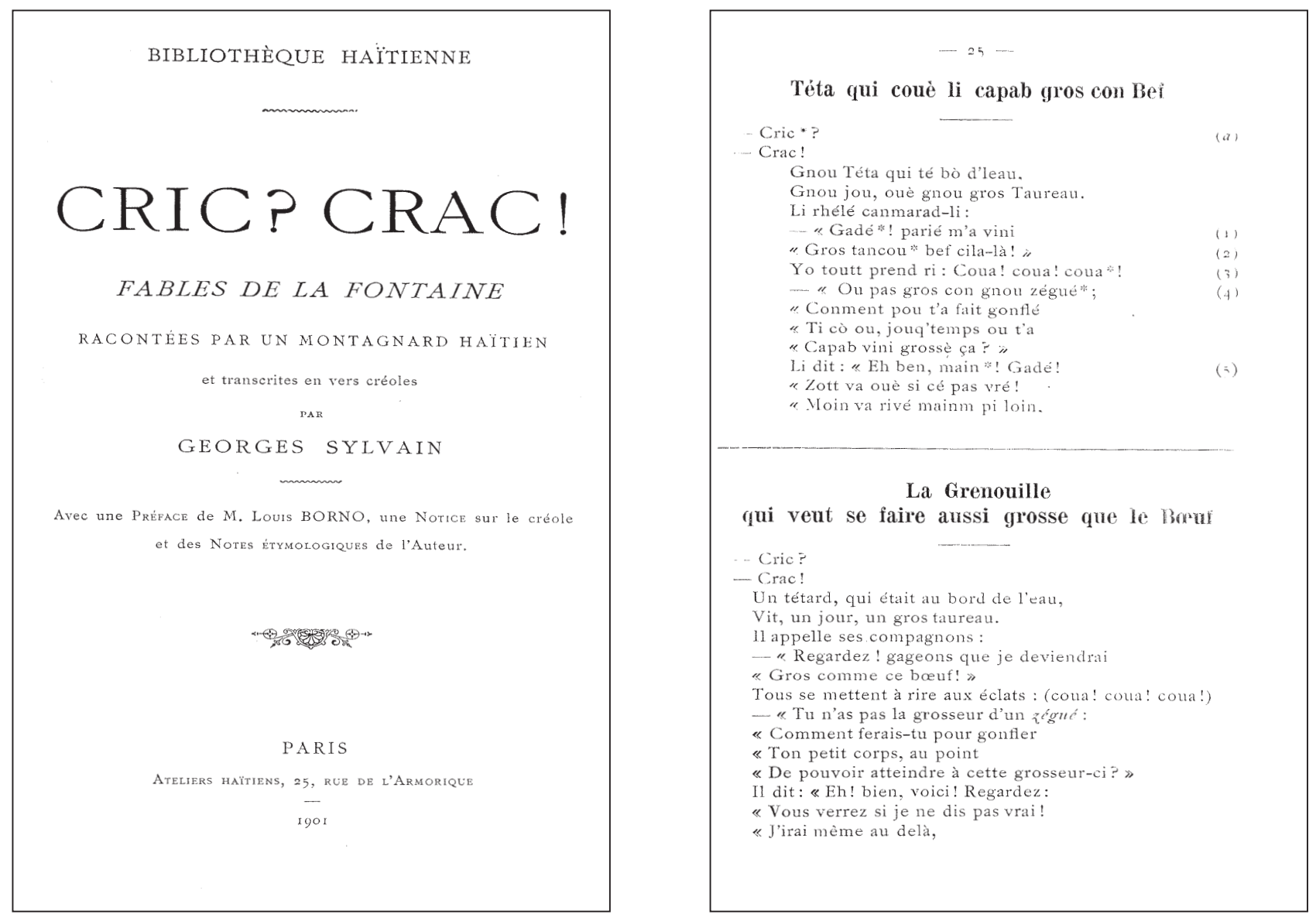

Fig. 1 : Cric ? Crac !, par Georges Sylvain

Comprendre comment de manière systémique se conçoit, s'écrit, se fabrique ce texte haïtien en quête d'une certaine lisibilité, mais aussi de légitimité (nationales et transnationales), peut apporter un éclairage unique sur la genèse des textes, plus particulièrement des traductions auctoriales, sans compter un apport au développement d'une génétique textuelle des littératures sans archives au sens strict du terme, comme celles d'Haïti. C'est là une tâche singulière à laquelle la critique (haïtienne ou non) doit s'atteler.

\section{Une génétique textuelle haïtienne, pour qui, comment ?}

D'un point de vue pragmatique, une approche génétique des textes haïtiens contribuera à l'enrichissement des études francophones en général, mais plus directement de la critique haïtienne qui bénéficiera de ces nouvelles recherches pour mieux analyser ses objets. Une telle entreprise contribuera également à la formation de chercheurs, tant en Haïti qu'à 
l'étranger, car il faudra mobiliser des équipes, les préparer à aborder les textes comme un processus, non comme une clôture bordant des messages politiques ou sociologiques à révéler. Sur ce plan, l'expérience de l'équipe de l'édition des œuvres de Rabearivelo trace déjà des voies prometteuses à poursuivre ${ }^{9}$, mais aussi à réévaluer. En effet, si l'écrivain malgache, comme les Haïtiens, a écrit dans deux langues, et pratiqué l'autotraduction, il reste que contrairement à un Frankétienne, son œuvre nous est parvenue massivement sous forme manuscrite plutôt qu'imprimée ; de plus, entre le malgache et le français, les interférences ou chevauchements sont plutôt rares, sinon inexistants. Ce sont là des différences significatives. Par ailleurs, nous ne disposons pas de manuscrits ou tapuscrits de Frankétienne avant les années 2000. L'auteur, dans la bonne tradition des $\mathrm{XVII}^{\mathrm{e}}$ et XVIII e siècles français et des écrivains haïtiens (les rares exceptions sont des écrivains du dehors), se faisait un devoir de les détruire, ou de s'en défaire. Il serait donc impossible d'établir un dossier génétique matériel de Dézafi et des Affres d'un défi ; par contre, il sera possible de reconstituer la méthode de production de ces textes, notamment du second pour lequel nous disposons de deux états imprimés de son hypotexte haïtien $(1975,2002)$. Travaillant dans une perspective plus ou moins similaire, Piat montre comment, à partir d'une lecture du texte imprimé, on peut reconstituer, du moins partiellement, sa genèse. En effet, soutient-il : « On peut faire l'hypothèse que certains phénomènes génétiques ont laissé des traces lisibles dans le texte imprimé et qu'il est possible, à partir de ces traces, de remonter au cœur du processus d'écriture ${ }^{10}$. » Il offre également dans son article des pistes d'analyse des traductions auctoriales, notamment sur le plan de la stylistique comparée, fort pertinentes pour une génétique des œuvres haïtiennes. Sa démarche ne serait pas sans rapport avec celle que d'autres appellent " génétique virtuelle », malgré toutes les réserves qu'on peut avoir sur cette appellation et la ou les recherches qui s'y rattachent, nous pouvons tirer profit des réflexions enclenchées entre autres par Patrick Dandrey et ses collaborateurs ${ }^{11}$.

D'autre part, notamment pour les textes qui nous intéressent ici au premier chef, le manque d'archives matérielles peut être comblé en partie par deux séries de matériaux linguistiques et discursifs préconstruits. Sur le plan phrastique, depuis les travaux d'Amédée Volcy et de Lucas notamment ${ }^{12}$, nous savons que le texte haïtien de Frankétienne se construit à partir de proverbes, dictons et autres syntagmes figés aisément repérables au niveau hypertextuel. Un exemple fort révélateur qui, du coup, nous permet de montrer un aspect particulier des livres de Frankétienne : leur " typo/topo/poéthique », dont un exemple est reproduit en figure 2 . Ici, il reprend graphiquement et scripturalement deux symbolismes haïtiens : l'un emprunté à la tradition vodou (la croix des carrefours et le cercueil de la douleur ou du malheur, sèkèymadoulè, dessinés sur la page); l'autre à un rituel profane, celui des bravades ou affrontements d'adolescents dont la formule d'ouverture est : «men kwa manman ou, men kwa papa ou, si ou pa pè vin pile $l »$ (voici la croix de ta mère et de ton père, si tu es brave traverse-la (ou plus littéralement, "viens la piétiner »), à laquelle fait suite un chorus de l'assistance, «Akasan men siro!" (l'acassan est prêt, voici le sirop ; autrement dit : le vin est tiré, il faut le boire). La mise en page complexe du texte haïtien, associant image, texte et typographie différentielle, n'est pas rendue dans le texte français, Les Affres d'un défi, qui banalise la matérialité de cette page à la fin d'un paragraphe :

La violence meurtrière de l'assassin attise notre soif de vengeance. Nos cicatrices bourgeonnent. Les dents du bourreau mordent impunément la chair de nos enfants. Qui aura

9. Voir Laurence Ink, Liliane Ramarosoa et Claire Riffard, Sauvegarde et valorisation des manuscrits malgaches: le cas de Jean-Joseph Rabearivelo. Un exemple de valorisation des manuscrits francophones, Paris, ITEM/EAC, 2010.

10. Julien Piat, «Figures et problèmes d'insertion dans la genèse de la trilogie de Samuel Beckett », Genesis, n²9, 2008, p. 44.

11. Voir Patrick Dandrey (dir.), Génétique matérielle, génétique virtuelle: pour une approche généticienne des textes sans archives, Québec, Presses de l'université Laval, 2009.

12. Voir Marie-Michèle Amédée, Les Proverbes en créole et dans le roman Dézafi : étude linguistique, sociologique et stylistique, Université de Montréal, Mémoire de maîtrise, 1982 ; Marie-Michèle Amédée Volcy, « Des Proverbes haïtiens dans Dézafi », Dérives, n 53-54, 1986/1987, p. 115-124 ; Rafael Lucas, "La littérarisation de la langue haïtienne », dans Jean Jonassaint, Typo/Topo/Poéthique sur Frankétienne, Paris, L'Harmattan, 2008, p. 123-146. 


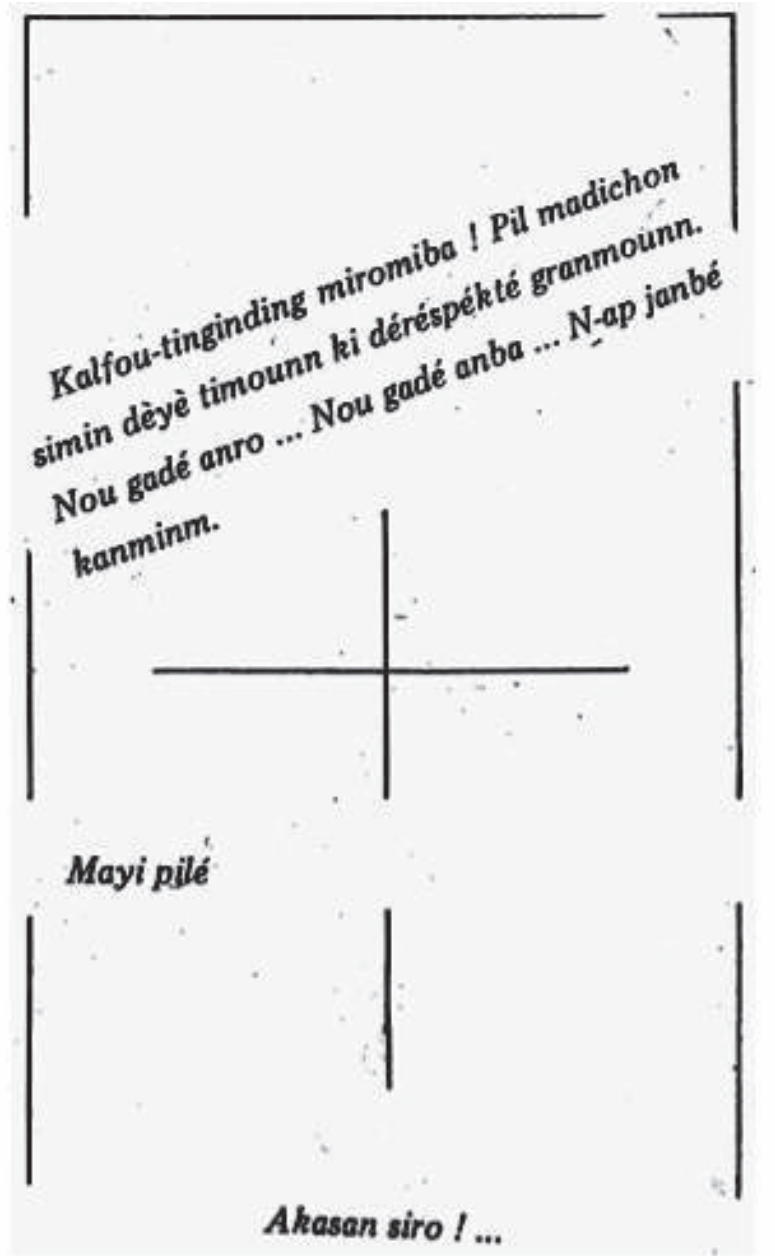

Fig. 2 : Frankétienne, Dézafi (1975), p. 281

su compter nos blessures [1] à ce dangereux carrefour de déséquilibre où tant d'infortunes pèsent sur nos épaules ${ }^{13}$ ? [2] Après avoir sondé les quatre horizons, nous sommes décidés à enjamber le fossé des vieilles malédictions, à briser la croix du malheur. [3] Nous avons pilé du maïs ; [4] immanquablement nous mettrons l'acassan au feu (Les Affres, p. 207-208) ${ }^{14}$.

Le texte haïtien de 2002 publié en France ne reproduit pas non plus la complexité visuelle de la première édition, mais propose une autre typo/topographie différentielle plus apte à faire sens pour un lecteur étranger (aux choses haïtiennes). En effet, sur une double page (voir fig. 3), nous avons en capitales corps 24 le texte de 1975 encadré de deux ajouts :

(a) Memwa nou / pran danse lwa / lan chak mak / zòtey nou trase / lan pousyè / lan chak twou / patpye nou fouye / lan chak vèvè / nou simen lan kalfou / pou lanmò / pa gen priz sou la vi 15 ;

(b) Baykou bliye, potemak sonje. Zodan konn mòde vyann, li pa konn konte mak ${ }^{16}$.

Memwa nou
pran danse lwa
lan chak mak
zòtèy nou trase
lan pousyè
lan chak twou
patpye nou fouye
lan chak vèvè
nou simen lan kalfou
pou lanmò
pa gen priz sou lavi.

KALFOU

TENGENDENG

MIWOMIBA.

PIL MADICHON SI-
MEN DEेYE TIMOUN KI
DERESPEKTE GRAN-
MOUN.
NOU GADE ANWO.
NOU GADE ANBA.
N AP JANBE KAN-
MENM.
MAYI PILE.
AKASAN SIWO !...
BAYKOU BLIYE, POTE-
MAK SONJE.
ZODAN KONN MÒDE
VYANN, LI PA KONN
KONTE MAK.

Fig. 3 : Frankétienne, Dezafi (2002), p. 270-271
13. Les passages soulignés sont ceux qui traduisent plus ou moins le texte haïtien dont nous numérotons les segments pour faciliter le repérage de leur traduction : «[1] Kalfou-tinginding miromiba! Pil madichon simin dèyè timounn ki déréspékté granmounn. [2] Nou gadé anro... Nou gadé anba...N-ap janbé kanminn. / [3] Mayi pilé /[4] Akasan siro !... » (Dézafi, p. 281).

14. Pour Les Affres d'un défi, nous citons toujours l'édition de 1979 ; par contre pour Dézafi nous nous référons soit au texte de 1975, orthographié, Dézafi, avec un accent aigu sur le «e », soit à celui de 2002 (fort différent d'abord par l'orthographe, mais surtout les nombreux ajouts et une nouvelle typo/topographie), orthographié Dezafi, sans accent, en conformité avec la graphie officielle de 1979.

15. Les barres obliques ne sont pas dans le texte; elles ne font qu'indiquer les retours à la ligne. Ce passage a été traduit assez fidèlement comme suit par Frankétienne : «[...] notre mémoire, chevauché par un loa sauvage, danse l'hystérie. Empreintes de nos orteils dans la poussière, trous creusés [...], vêvês tracés à la croisée des chemins, pour que la mort ne l'emporte jamais sur la vie » (Les Affres, p. 207).

16. Notre traduction : «Qui frappe oublie, qui subit s'en souvient. La dent mord la chair, elle ne compte pas les marques. » 
Le premier ajout, à une suppression près - «nan labou » qui suivait «pat-pié nou fouyé » (Dézafi, p. 280) -, n'est que la dernière phrase du paragraphe initial précédent découpée en vers composés en italique corps 24 ; le second est emprunté au texte français de 1979 qui explicite le graphisme de 1975 en ces termes : «ce dangereux carrefour de déséquilibre »; " la croix du malheur ». L'addition tirée des Affres d'un défi montre le va-et-vient de plus en plus important entre les deux langues (haïtien et français) dans la production des textes de Frankétienne, à partir des années quatrevingt, notamment son théâtre ${ }^{17}$. Ici, en effet, nous avons sous une forme sentencieuse, calquant la syntaxe du proverbe haïtien qui le précède («Baykou bliye, potemak sonje »), une phrase énigmatique qui traduit l'esprit de l'ajout du texte français cité intégralement plus haut : « La violence meurtrière de l'assassin attise notre soif de vengeance. [...] Qui aura su compter nos blessures [...] ? » (Les Affres, p. 208).

Il importe de noter que le texte français a recours à l'interrogatif pour marquer la question, le doute, alors que le texte haïtien emploie une forme affirmative proverbiale pour susciter l'énigme : «zodan konn mòde vyann, li pa konn konte mak». De plus, cette forme sentencieuse haïtienne exige du lecteur un savoir extralinguistique (partagé avec l'écrivain ou le locuteur) pour être comprise, alors que le texte français ne le demande pas puisqu'il est explicite. Aussi, ne pourrait-on pas se demander si les rhétoriques fort différentes des textes haïtien et français ne sont pas liées aux spécificités des deux langues ?

En effet, sur le plan strictement textuel, le texte français ne rend pas la concision presque « sentencieuse » de l'haïtien. La réécriture en français tend plutôt vers l'explication, comme le montre, entre autres, une comparaison du deuxième paragraphe des Affres (1979) avec celui de Dézafi (1975). D’un premier ensemble de quinze phrases totalisant cinquante-trois mots, nous passons à un second de cent quarante mots regroupés en vingt-sept phrases, dont douze générées à partir de la liste de verbes formant la phrase initiale : "Dòmi lévé gadé maché manjé lanbé taté souflé tonbé kouri ralé jounin grangou. » Plutôt que de traduire littéralement («Dormir se lever regarder marcher manger lécher tâter souffler tomber courir endurer une journée de faim »), Frankétienne transforme chaque verbe en générateur de récit d'une phrase, sauf «maché » qui en génère deux :

\begin{abstract}
Dormir avec l' espoir que la lumière drainera nos angoisses nocturnes. Se réveiller loin des songes désentravés, le corps enlépré de solitude. Regarder l'immensité des déserts inarpentés. Errer à travers la meublerie des désirs. Remuer le ciel et la terre jusqu'au saignement des étoiles et des pierres. S'empiffrer de nourriture. Lécher d'appétit. Palper avec prudence. Souffler sur des morceaux brûlants. Choir/déchoir. Fuir à toutes jambes. Crever de faim des jours entiers (Dézafi, p. 11 ; Les Affres, p. 2).
\end{abstract}

Un autre exemple qui nous porte à soutenir l'hypothèse d'une spécificité des deux langues induisant des stratégies d'écriture différentes est le passage du français à l'haitien analysé plus haut (Les Affres, p. 207-208 ; Dezafi, p. 271). De quatre phrases françaises plutôt explicatives de trente mots, Frankétienne aboutit à deux dictons haïtiens, ou pris comme tels, le deuxième n'étant qu'une invention d'auteur, qui font treize mots. Cette concision de l'expression haïtienne est tout à fait conforme au génie de cette langue dite andaki, qui tend plus vers l'énigmatique ou le ludique que l'explicatif ou la clarté, contrairement au français, du moins dans l'usage qu'en font Frankétienne ou d'autres écrivains haïtiens.

La dimension typo/topo/graphique marginale dans Dézafi et Les Affres d'un défi deviendra de plus en plus importante à partir de L'Oiseau schizophone (1993) pour atteindre un sommet avec H'Éros-Chimères (2002). Le travail d'écriture ne relève plus du manuscrit ni même du tapuscrit, car il y a fusion entre ce qui serait de l'ordre du manuscrit/tapuscrit/montage et du domaine du livre imprimé. En ce sens, même dans les cas d'ouvrages pour lesquels nous disposons de manuscrits, l'écart entre ces derniers et les livres imprimés est si grand, qu'il est probablement vain de chercher des traces matérielles de typo/topo/graphie antérieures à la page imprimée. Celle-ci relève d'un astucieux bricolage manuel et informatique,

17. Lire le témoignage de Jenny Leignel sur l'écriture et la production de Totolomannwèl dans nos notes sur cette pièce de Frankétienne, dans Typol Topo/Poéthique, op. cit., p. 336-338. 
travail de collaboration étroite de l'auteur notamment avec sa femme typographe, dont seul le résultat compte. C'est ce que semble montrer la double page analysée plus haut. Aussi, nous pouvons avancer l'hypothèse que la suppression du syntagme «nan labou » a plus à voir avec des contraintes typographiques que langagières. En effet, si nous ajoutons ce syntagme nous créons une ligne supplémentaire qui ne permet plus d'avoir le blanc entre la phrase versifiée et ce qui suit. De plus, on risque de détruire la composition presque en pavé de la double page. L'alignement des vers à pleine hauteur sur les deux pages ne serait plus possible, et l'on aurait immanquablement un vers perdu sur une troisième page. Réduire le corps des caractères pourrait être une solution, mais on perdrait le sentiment de plein, sinon de trop-plein, d'affrontement angoissant que tente de signifier le dispositif typo/topo/graphique qui redouble et renforce l'énoncé linguistique. Plus que pour tout autre écrivain francophone, la typographie est signifiante pour Frankétienne. Elle participe à la production du sens même dans l'œuvre, et joue incontestablement un rôle important dans la genèse ${ }^{18}$.

Il est donc évident que l'édition génétique de ces ouvrages (Dézafi (1975)/Les Affres d'un défi (1979)/ Dezafi (2002) dans un tout raisonné et cohérent demande un dispositif typo/topo/graphique particulier dont nous donnons en annexe une première ébauche qui permet du même coup au lecteur intéressé de mieux saisir le travail de réécriture de l'auteur, qui est d'abord une amplification-explication. Mais, étrangement, les ajouts les plus massifs sont dans le texte haïtien de 2002, qui tente d'atteindre un public étranger, donc obligé d'intégrer tout en respectant la rhétorique énigmatique haïtienne, des exigences explicatives du texte français de 1979 dédié spécifiquement à un public transnational.

Enfin, sur le plan narratologique ou discursif, une génétique haïtienne tirera profit des récits oraux ou écrits populaires, littéraires ou « savants », sur lesquels se construisent en tout ou en partie les spirales de l'écrivain. Par exemple, la résurrection des zombis par le sel est non seulement un récit populaire fort répandu en Haïti, mais il est entré depuis les années vingt et trente dans le discours anthropologique et artistique international, notamment avec le livre de William Seabrook, Magic Island (1929), l'une des sources du film White Zombie de Victor Halperin (1932). Ce récit mythique est aussi repris par Alfred Métraux avec une certaine distance critique, dans Le Vaudou haïtien (1958). Plus qu'une source d'inspiration mythique, il est une compétence narrative que l'écrivain peut actualiser avec des variations idiosyncratiques. Mais il a une syntaxe propre, comme un passage obligé avec ses grandes étapes : avant la zombification (du sujet) / sa traversée vers l'autre monde / l'univers du ou des zombis / leur libération (par le sel) / après la libération. Plus concrètement, ce récit dans sa double relation architextuelle et hypertextuelle avec le texte de l'écrivain (ou avec toute autre énonciation, dont celle du conteur haïtien), se décompose invariablement comme suit : (1) mort apparente d'un jeune homme prétentieux ou d'une jeune fille hautaine ; (2) traversée du défunt déterré de sa ville pour effrayer la population et réaffirmer la toute-puissance du hougan empoisonneur ; (3) arrivée sur la plantation du maître pour le rituel de zombification ; (4) rappel du régime alimentaire strict et des règles draconiennes de travail et de soumission ; (5) trahison ou erreur d'une femme qui donne le sel au(x) zombi(s) ; (6) libération du ou des zombis de l'emprise du maître, la révolte vengeresse ou la disparition. C'est ce scénario archiconnu que Frankétienne monte (comme un film) parallèlement à d'autres microrécits populaires : de l'exode rural incarné par Gaston et Rita jusqu'à l'exil intérieur de Jérôme à la campagne en passant par l'épisode rocambolesque du pasteur Pinechrist. Il constitue un état premier ou élémentaire, spéculatif certes, du «scénarique19 »de Dézafi, qui pourrait être complété en puisant notamment dans l'imposant corpus d'entrevues auctoriales sur ces œuvres, en plus des nombreux passages métadiscursifs ou poéthiques de Mûr à crever (1968) et Ultravocal (1972), qui peuvent nous éclairer sur la méthode créative

\footnotetext{
18. Un autre exemple est donné et commenté en annexe, à propos de la réécriture de «palé dépalé » (1975) en «Pale/depale » (italique corps 24), voir la note 5 de l'annexe.

19. Voir Henri Mitterand, «Sur le "scénarique" », Genesis, n 30, « Théorie : état des lieux », 2010, p. 69-84.
} 
de l'écrivain, qui rappelait dans une entrevue avec Ulrich Fleischmann :

Je crois qu'il y a des liens très étroits au niveau linguistique d'abord, entre Ultravocal et Dézafi. J'ai pu bénéficier des apports linguistiques de l'écriture d'Ultravocal en rédigeant Dézafi. Sur ce point, je n'ai pas senti de barrières entre les deux langues 20 .

La démonstration ici faite à partir des œuvres de Frankétienne pourrait être reprise presque terme à terme pour les récits de Depestre, notamment Hadriana dans tous mes rêves (1988) qui peut être lu comme un clin d'œil au diptyque Dézafi/Les Affres d'un défi. En effet, ce roman reprend non seulement le récit de la résurrection du zombi, mais aussi celui du hougan tout puissant jaloux, qui échoue dans sa vengeance. En fait, fort probablement, sur le plan des principes, si l'on accepte l'hypothèse, ailleurs diversement exprimée, du texte francophone comme hypertexte allogène d'un hypotexte indigène, autrement dit, jusqu'à un certain point traduction d'un texte indigène 21 , une telle analyse s'appliquerait à l'ensemble du corpus narratif haïtien et poserait les bases de toute étude génétique de ce corpus.

20. Ulrich Fleischmann, « Entrevue avec Frankétienne sur son roman Dézafi », Dérives, n 7, 1977, p. 18.

21. Voir entre autres : Chantal Zabus, The African Palimpsest: Indigenization of Language dans the West African Novel, New York et Amsterdam, Rodopi, 1991, 2nd enlarged edition, 2007 ; Nora-Alexandra Kazi-Tani, Roman africain de langue française au carrefour de l'écrit et de l'oral : Afrique noire et Maghreb, Paris, L'Harmattan, 1995 ; Jean Jonassaint, Des romans de tradition haïtienne, op. cit. 


\section{Annexe \\ Ébauche d'édition génétique d'un extrait de Dézafi/ Les Affres d'un défi de Frankétienne}

Extrait des premières pages (7 à 10) de Dezafi (2002) édité et analysé en comparaison avec le texte de 1975 et la version française correspondante de 1979 (Les Affres d'un défi, 1-2) pour montrer concrètement et visuellement, entre autres, un aspect majeur du travail de Frankétienne : l'amplification de son texte par dérivation ou redoublement à partir d'un mot, d'une phrase, d'un paragraphe pour des fins d'explication ou de clarification, mais aussi par simple plaisir d'explorer les limites d'une langue. L'amplification peut se faire typo/ topographiquement (augmentation du corps des caractères, soulignements en italique, gras, capitales) ; scripturalement par des ajouts massifs ou non, par allitérations de suffixes ou préfixes, oppositions sémantiques, développements narratifs ou discursifs à partir d'un mot, listes paradigmatiques de mots ou catégories du discours (verbe, adjectif, adverbe), explications synonymiques ou définitionnelles...

Globalement, à partir de la première phrase de l'incipit de 1975, le texte se déploie autour des cinq sens. Cette micro-perspective sensorielle n'est pas sans rappeler L'Espace d'un cillement de Jacques Stephen Alexis (1959) dont chaque partie ou «mansion », sauf la « coda » finale et la sixième «mansion » titrée «Le sixième sens », est désignée par un sens (la vue, l'odorat, l'ouïe, le goût, le toucher) qui permet aux principaux personnages, El Gaucho et la Niña, d'un bordel de Port-au-Prince de remonter leur mémoire cubaine et leur traversée de la Caraïbe pour se retrouver.

Les passages en corps 11.5 sont de 1975, sauf les mots soulignés qui sont des ajouts mineurs ou des variantes orthographiques allant au-delà des différences évidentes entre les " graphies» de 1975 et 2002 ; ceux en corps 14 sont les ajouts massifs de la réécriture de 2002. Sur la page de droite, nous reproduisons la réécriture en français de 1979 (pour l'instant, à part les caractères et les mises en page différents d'une édition à l'autre, nous n'avons trouvé aucun changement, du moins significatif). Quand nécessaire, nous signalons les écarts de transformation/traduction du texte de 1975 ; et nous offrons notre propre traduction française pour les ajouts de 2002, tentant, dans la mesure du possible, de rester au plus près de l'haïtien avec ses phrases elliptiques ou infinitives courantes, du moins conformes au génie de cette langue. 
[a] Branchbwa makònen lan fon yon vye lakou kote kretyenvivan (1) pase raman.

Depi ou pwoche lan viwonn lakou saa, lapèrèz anpare ou. Bridsoukou vant ou tanmen bouyi. Ou pran latranblad lasengi. Tranblemannkè senvlenvlen mafyengen dekrenmen mazenflen sendenden.

$[\ldots]^{2}$

[b] Lan fon lakou bazawa, yon ponyen sèl koumanse (3) fonn lan yon katafal bonm dlo cho. Yon bonm degrade, kolboso toupatou, nwa anba kouch lafimen. Lan mitan yon boukandife, yon latriye grennsèl tanmen pete. Lavi ak lanmò pa janm sispann twoke kòn.

[c] Dòmi leve gade rele mache manje lanbe niche tate soufle tonbe kouri joure babye rale jounen grangou. (4)

$$
\begin{aligned}
& { }_{\text {[d] Pale/depale. }{ }^{(5)}} \text { Lang lou. } \\
& \text { Lang koupe } \\
& \text { miyetmoso.(6) }
\end{aligned}
$$

1 Dans l'édition de 1975, nous lisons : «vivan dé pié », forme francisée non courante, littéralement presque, (être) vivant (à) deux pieds, qui devient : « Kretyenvivan » (chrétien vivant), expression haïtienne populaire pour nommer un être humain. Ici, manifestement, la substitution tend vers plus d'authenticité ou de lisibilité, tendance assez marquée dans les réécritures de Frankétienne.

2 Nous reproduisons ici que le premier paragraphe de la page d'ouverture de l'édition de 2002 de Dezafi, et coupons entièrement la page 8 qui suit : trois paragraphes totalisant 153 mots qui constituent de loin la part la plus importante de l'amplification de la réécriture des deux premiers paragraphes du texte de 1975 .

3 Variante phonologique et orthographique « kòmansé » devient « koumanse » (commencer). Cette substitution n'implique aucun changement sémantique ni même stylistique réel, «koumanse » n'étant qu'une variante sociolectale de « kòmansé ».

4 Noter qu'il n'y a plus de blanc entre les deux paragraphes comme dans la première édition. De plus, le deuxième paragraphe de 1975 est segmenté en divers petits paragraphes avec entre autres le passage qui suit (souligné en gris par nous) formant un bloc particulier isolé par deux espaces blancs assez importants.

5 L'introduction de la barre oblique ici tend à renforcer l'opposition entre «pale » (parler) et (« epale » (déparler). C'est un ajout (de ponctuation) pour clarifier l'expression originale : «palé depalé ». 6 Le changement ici est, d'une part, typo/topographique, corps et style du caractère sont beaucoup plus gros, mais aussi la disposition du fragment sur la page qui se présente sous forme de vers (édition 2002) plutôt que de prose (1975); d'autre part, orthographique, « miyètmoso » qui s'écrit en un mot plutôt que deux avec un tiret, " miyèt-moso ». Dans la réécriture de 2002, Frankétienne tend à s'aligner plus sur la logique de la langue haïtienne, et beaucoup moins sur celle de l'orthographe française. Par ailleurs, ce passage en italique corps 24 montre que même ayant accès au manuscrit ou un état imprimé premier, il nous sera difficile, sinon impossible, de restituer concrètement ou matériellement le processus de la mise en forme typo/ topographique qui est relativement importante ici, perdu dans la suite des (plausibles) essais et erreurs (virtuels) à l'écran. C'est là une difficulté particulière au texte de Frankétienne, il nous pousse jusqu'à un certain point vers une démarche plus ou moins spéculative. 
Réécriture (traduction ou adaptation) en français par Frankétienne du texte de 1975 dans Les Affres d'un défi (1979). Quand il y a un ajout significatif dans le texte haitien de 2002 que nous reproduisons, nous le signalons par notre traduction soulignée entre crochets ; de même quand le texte français (1979) s'écarte significativement du texte haïtien (1975) nous donnons également notre traduction. Pour faciliter la lecture, nous ne respectons pas la mise en page de l'ouvrage de 1979, mais suivons plutôt la segmentation des paragraphes dans l'édition de 2002 de Dezafi.

[a] Enchevêtrement de branches d'arbres au fond d'une vieille cour fréquentée rarement par des êtres humains (Les Affres 1).

[Dans le texte de 2002, cette phrase est suivie par trois autres qui explicitent les sentiments d'épouvante éprouvés face à cette cour macabre : " Dès que tu t'approches près de cette cour, la peur s'empare de toi. Du coup ton ventre se met à bouillir. Tu trembles d'une danse de saint-guy. Palpitations cardiaques saint-glinglin mal au point déplumé écrémé gourmandé saint-linlin. » Suite à ce passage la cour infernale est décrite longuement sur trois paragraphes de 153 mots, non reproduits ici, à partir de termes liés aux cinq sens - vue (yeux), ouïe (oreilles/ voix), odorat (nez), goût (langue/ bouche), toucher (peau) — qui deviennent des générateurs de phrases. Il y a une amplification du sentiment de peur exprimé dans ce paragraphe initial et une prédominance des termes reliés au toucher, à la vue et à l'ouïe notamment : « zòrèy » (oreilles), « alekout » (à l'écoute), « tande » (entendre), 《 satouyèt » (chatouiller), « toutkoulè » (tout couleur », « frisonnen » (frissonner), «mizik » (musique), « vwa » (voix), «tresayi » (tresaillir), « chèdepoul » (chair de poule), « voye je gade » (jeter un coup d'œil), « lafimen » (la fumée), « vant plen » (le ventre plein), «swaf dlo » (soif d'eau), « frape » (frapper), « vantdeboutonnen » (la ceinture ouverte, [manger] à volonté, à l'excès).]

[b] [Au fond d'une cour dangereuse,] Une poignée de sel commence à se dissoudre dans un [immense] chaudron d'eau bouillante. Un chaudron abîmé, complètement bosselé, noirci de couches de fumée. Au milieu d'un feu de bois, d'innombrables grains de sel crépitent. Incessant combat entre la vie et la mort (Les Affres 1).

[c] Dormir avec l'espoir que la lumière drainera nos angoisses nocturnes. Se réveiller loin des songes désentravés, le corps enlépré de solitude. Regarder l'immensité des déserts inarpentés. Errer à travers la meublerie des désirs. Remuer le ciel et la terre jusqu'au saignement des étoiles et des pierres. S'empiffrer de nourriture. Lécher d'appétit. Palper avec prudence. Souffler sur des morceaux brûlants. Choirl déchoir. Fuir à toutes jambes. Crever de faim des jours entiers (Les Affres 2).

[La traduction plus ou moins littérale de la phrase devrait se lire comme suit : « Dormir se lever regarder crier marcher manger lécher flatter tâter souffler tomber courir grogner injurier endurer une journée de faim. »]

[d] Parler sans cesse. Déraisonner. Avoir la langue engourdie ou cisaillée en mille morceaux (Les Affres 1). 
[e] Vant plen. Vant vid. (1) Trip kòde. Swaf dlo. Swaf lanmou. Anvi solèy. Anvi limyè. Reve zetwal. Reve fanm siwolin. Reve boul dayinaflo k ap woule sanrete lan mèvèy boulozay. Reve [p. 9] vwayaj lasirèndyaman $\mathrm{k}$ ap file kannale sou lanmè jouk aziboutou. Imajinen degenn lakansyèl jouk lòtbò zilepadokay.

[f] Abiye banda. Abiye anreleng. Kouche mòksis. Leve kè (2) kontan. Griyen dan. File grandou lan douvanjou. Frape tanbou sou konpa brennzeng. Danse vantdeboutonnen.

[g] Pwonmennen bouloze toutouni. Vlope lan ranyon. Gaye lan (3) lanmou tètkale bounda-ouvè. $\left(^{4}\right)$ Anfouye lan madigra rabòday maskawon. Anfouraye lan deblozay lanmò. Anfoudwaye lan kagoulaw. (5) Kilès pami nou $\mathrm{k}$ ap viv toutbon ? Kilès ?

1 Cette seconde phrase est un ajout. Elle contraste avec la première, et donne plus de sens à ce qui suit : « Trip kòde » (littéralement : tripes cordées dans le double sens d'emmêler et d'amarrer) qui marque le manque de nourriture («vant vid», ventre vide) et autre manque ou soif d'eau, d'amour, de soleil, de lumière, d'étoiles, de femmes, etc.

2 Au prime abord, les syntagmes «Levé kontan » (1975) et « Leve kè kontan » (2002) expriment le même sentiment, sans nuance ou presque. Mais, il reste qu'il est difficile de décider si l'écrivain cherche à exprimer une nuance, ou simplement renforcer le sentiment de contentement dans sa phrase ? En effet, en haïtien « kè Kontan » (littéralement : cour content) peut être « le cœur content », « le contentement », « l'euphorie », « heureux, euphorique », ou la phrase elliptique « M kè kontan » (je suis content). Ces indécisions sont courantes en haïtien, notamment dans le parler dit « andaki » (dire une chose pour exprimer une autre). Frankétienne en tire profit tant dans ses textes français qu'haïtiens. Un bon exemple est sa traduction de « leve [kè] kontan » («Se lever en pleine euphorie ») qui peut se lire doublement : se lever euphorique/ se lever dans une euphorie (qui n'est pas nécessairement nôtre).

3 Substitution phonologique de «nan » par «lan ». Les deux formes sont courantes ayant la même valeur prépositionnelle du « dans » français.

4 La réécriture en français de 1979 du texte « original » semble avoir influencé celle de 2002 en haïtien. En effet, «Se fourvoyer dans de folles amours » n'est pas une traduction terme à terme de « gaye nan lanmou », par contre elle rend bien l'esprit, sinon la lettre, de « gaye lan lanmou tètkale bounda-ouvè », une formule assez « grossière » qui ne se retrouve pas dans le parler quotidien. En effet, l'expression « tètkale bounda-ouvè » (littéralement la tête rasée, le cul ouvert, autrement dit sans retenue) forme un oxymoron avec « lanmou » (amour) qui en haïtien est une forme très courtoise qui renvoie plutôt au sentiment qu'à l'acte plutôt grossier qu'évoque « bounda-ouvè ». Ce va-et-vient ou aller-retour haïtien/ français et vice versa serait à étudier plus à fond.

5 Les passages soulignés sont des ajouts qui permettent à Frankétienne de donner un autre rythme à ce fragment du paragraphe original, entre autres, par l'allitération du préfixe « $\mathrm{AN}$ », des suffixes « $\mathrm{YE} »$ et « $\mathrm{AY} »$, en plus des variations sonores que permettent les mots — Anfou/ ye [...]. Anfoul Raye [...]. Anfou/ Dwaye — dont les deux premiers, « anfouraye » et « anfouye », seraient des synonymes selon le Haitian-English Dictionary de Bryan Freeman et Jowel Laguerre (Lawrence et Port-au-Prince : Institute of Haitian Studies, University of Kansas, 2002). 
[La traduction littérale de ce passage devrait se lire comme suit : « Parler/ déparler. Langue lourde. Langue coupée en miettes. » Amplification pour bien ancrer le sens (faire ressortir / jaillir le sens).]

[e] Etre repu. Avoir les tripes encordées par la douleur. Éprouver une soif d'enfer (Les Affres 1).

[Dans la réécriture de 2002, ces trois phrases sont suivies de neuf autres infinitives, le verbe " avoir » étant sous-entendu dans les quatre premières : «Soif d'amour. Envie d'amour. Envie de soleil. Envie de lumière. Rêver d'étoiles. Rêver de femmes suaves. Rêver d'un numéro extra telle une boule magique qui roule dans un champ de merveilles excentriques. Rêver de voyage de Sirène diamantée qui file à toute vitesse sur la mer jusqu'au bout du monde. Imaginer la démarche d'arc-en-ciel à l'autre bord d'îlesans-toit » (Dezafi 9-10).]

[f] Se parer comme un paon. [S'habiller misérablement.] Se coucher de mauvaise humeur. Se lever en pleine euphorie. Rire à pleines dents (Les Affres 1-2).

[Dans la réécriture de 2002, ces trois phrases sont suivies de trois autres infinitives qui renforcent l'idée d'euphorie, de plaisir : « Hisser l'immense cerfvolant au devant-jour. Battre le tambour au compas survolté. Danser à l'excès. »]

[g] Marcher [joyeusement] tout nu ou recouvert de haillons. Se fourvoyer dans de folles amours. [Se perdre dans la sensualité lascive de la danse carnavalesque.] S' enliser dans [le tumulte de] la mort. [S'enfoncer dans la cagoularde.] Mais qui parmi nous vit réellement? Vraiment, qui ? (Les Affres 2). 
Jean Jonassaint, Professeur de littératures francophones à Syracuse University, coresponsable avec Yolaine Parisot du projet d'édition génétique d'œuvres haïtiennes au sein de l'équipe « Manuscrit francophone » de l'ITEM, est auteur de plusieurs travaux sur la production et la réception des textes, notamment La Déchirure du (corps)texte et autres brèches (Dérives/Nouvelle optique, 1984) ; Le Pouvoir des mots, les maux du pouvoir. Des romanciers haïtiens de l'exil (PUM/L'Arcantère, 1986) ; Typo/Topo/Poéthique sur Frankétienne (L'Harmattan, 2008). Il complète actuellement une édition critique de La Tragédie du Roi Christophe, à paraître dans Césaire, Euvres complètes, Paris, CNRS Éditions, coll. «Planète Libre », 2013.

Jean Jonassaint, jjonassa@syr.edu

\section{Des éditions génétiques haïtiennes : Pourquoi ? Pour qui ? Comment ?} Le cas de Dézafi/Les Affres d'un défi de Frankétienne

Partant des questions de Sartre dans Qu'est-ce que la littérature?, cet article met en lumière le Pourquoi, le Pour qui, et le Comment d'une entreprise d'édition génétique d'œuvres haïtiennes, plus particulièrement de Dézafi/Les Affres d'un défi de Frankétienne. En effet, pour la période contemporaine, ces œuvres sont fort caractéristiques des productions littéraires haïtiennes avec leur double tension : d'une part, linguistique langue de la majorité de la population (l'haïtien)/langue principale des productions littéraires (le français) - ; d'autre part spatiale - l'ici de la représentation/l'ailleurs des procès de production et de consécration. Enfin, sur le plan théorique, il montre comment une telle recherche peut contribuer à enrichir la génétique textuelle, notamment sur le plan des traductions auctoriales et de la stylistique comparée.

Taking as its starting point Sartre's questions in What is Literature?, this article highlights the "Why", the " "For whom", and the "How" of a genetic edition of Haitian works, in particular [of] Frankétienne's Désafi/Les Affres d'un défi. Indeed, these works are characteristic of contemporary Haitian literature. At play here is a twofold pressure: on the one hand a linguistic tension, the majority of the population's language (Haitian) vs. the main language of literary productions (French), on the other, a spatial tension, the Here of representation vs. the Elsewhere of production and recognition processes. Finally, on a theoretical level, this article shows how such an analysis can enrich textual genetics, in particular for auctorial translations and compared stylistics.

Ausgehend von den Fragen Sartres in Qu'est-ce que la littérature? beleuchtet dieser Beitrag das Was, das Für wen, und das Wie einer Unternehmung genetischer Ausgaben haitischer Literatur insbesondere von Dézafi/Les Affres d' un défi von Frankétienne. In der Tat sind diese Werke, was die zeitgenössische Periode betrifft, sehr charakteristisch für die haitische, literarische Produktion, mit ihrer doppelten Spannung: zum einen sprachlich - die Sprache der Bevölkerungsmehrheit (das Haitische)/Hauptsprache der literarischen Produktion (das Französische) - ; zum anderen räumlich - das Hier der Darstellung/das Woanders der Produktions- und Bestätigungsprozesse. Schließlich zeigt sich auf theoretischer Ebene, wie solche Forschungsarbeit bereichernd zu einer Textgenetik beitragen kann, insbesondere auf der Ebene auktorialer Übersetzung und vergleichender Stilistik.
Partiendo de las preguntas que Sartre plantea en ¿Qué es la literatura?, este artículo estudia el Por qué, el Para quién y el Cómo de una edición genética de obras haitianas, y más particularmente de los textos Dézafi y Les Affres d'un défi de Frankétienne. En efecto, estas obras son muy características de las producciones literarias del periodo contemporáneo, con su doble tensión: por una parte, lingüística -idioma de la mayoría de la población (el haitiano)/idioma principal de las producciones literarias (el francés)-, por otra parte, espacial -el aquí de la representación/el allá de los procesos de producción y consagración. Finalmente, este estudio demuestra, a nivel teórico, hasta que punto una investigación de este tipo puede contribuir a enriquecer la genética textual, en particular, en lo que se refiere a las traducciones autorales y a la estilística comparada.

A Partire dalle questioni di Sartre in Qu' est-ce que la littérature?, questo articolo mette in luce il Perché, il Per chi, et il Come di un'iniziativa di edizione genetica di opere haitiane, più particolarmente di Dézafi/Les Affres d'un défi di Frankétienne. In effetti, per il periodo contemporaneo queste opere sono emblematiche delle produzioni letterarie haitiane, con la loro duplice tensione: da una parte linguistica - lingua della maggioranza della popolazione (l'haitiano)/lingua principale delle produzioni letterarie (il francese); dall'altra spaziale - il qui della rappresentazione/l'altrove dei processi di produzione e consacrazione. Infine, sul piano teorico si mostra come tale ricerca possa contribuire a arricchire la genetica testuale, propriamente delle traduzioni d'autore e della stilistica comparata.

Com base nas perguntas de Sartre em Qu'est-ce que la littérature ?, este artigo põe em destaque o Porquê, o Para quem, e o Como de um empreendimento de edição genética de obras haitianas, mais particularmente de Dézafi/Les Affres d'un défi de Frankétienne. Com efeito, para o período contemporâneo, estas obras são muito características das produções literárias haitianas com a sua dupla tensão: por um lado, linguística - língua da maioria da população (o haitiano)/langue principal das produções literárias (o francês) -; por outro lado, espacial - o aqui da representação/o outro lugar dos processos de produção e de consagração. Por último, no plano teórico, ele mostra como essa investigação pode contribuir para enriquecer a genética textual, nomeadamente no plano das traduções autorais e da estilística comparada. 